\title{
Acupuncture with Planning Treatment According to Diagnosis: A Trial Study of Protocol and its Effects for Stiff Shoulder
}

\begin{abstract}
Background: In order to standardize an effective acupuncture therapy for chronic dull pain and stiffness of neck and shoulder, we made a protocol characterized with planning treatment according to diagnosis and examined its availability.

Methods and findings: Thirty-two patients were categorized into three groups based on the pathophysiology of Traditional Chinese Medicine: Blood stasis due to Qi stagnancy (Group-1), Deficiency of Liver-blood (Group-2), Yin deficiency of Liver and Kidney (Group-3) according to their subjective symptoms and signs. Acupuncture treatment was performed once a week over a two week period in accordance with the protocol that was arranged to each group. The averaged VAS values after the $3^{\text {rd }}$ therapy in the whole groups decreased significantly $(p<0.01)$ as compared with that of the initial visit before therapy. Investigating each group, the averaged VAS value after the $3^{\text {rd }}$ therapy in Group 1 was significantly $(p<0.05)$ lower than that of the initial visit at baseline. In other groups, although the VAS scores showed a gradual decrease according to the treatment, there was unfortunately no significance in the changes of the VAS. There were no side effects in all the treatment of this study.
\end{abstract}

Conclusions: These results indicate that the present study protocol would be effective in relieving the patient's subjective symptoms, and it would alternatively provide an effective and safe medical treatment for stiff neck and shoulder, although it should be modified for Groups 2 and 3.

Keywords: Acupuncture - Protocol - Shoulder stiffness - Neck pain

Submitted: 12 November 2017; Accepted: 25 November 2017; Published online: 30 November 2017

\section{Introduction}

The shoulder is the most mobile joint in the body and shoulder pain is an important medical and socioeconomic problem in the western world, with between $7 \%$ and $26 \%$ of the population reporting shoulder problems at any one time [1]. By contrast, chronic dull pain and stiffness of the neck and shoulder is called Katakori in Japanese, which ranks first among symptoms that the women show in Japan and is second among men based on the national livelihood survey results performed in 2015 by the Ministry of health, labor and welfare [2]. In some cases, symptoms such as headache, nausea, neck pain, shoulder pain and/or arm pain might accompany, and be considered a reason for the early symptom of cervical-omo-brachial syndrome as well $[3,4]$. A local blood circulatory disturbance on the trapezius and its neighbouring muscles possibly is raised due to muscular sustained strain and a consequential lack
Wenping Zhang ${ }^{*, 1,2}$, Kaito Mizuno', ${ }^{1,2}$ Masayuki Kanehara ${ }^{3}$, Ken Takagi ${ }^{2,4}$, Ryohei Ishiyama ${ }^{5}$, Shigeru Urata ${ }^{1,2}$, Ko Nishimura $1,2,4$

${ }^{1}$ Faculty of Acupuncture \& Moxibustion, Suzuka University of Medical Science (SUMS), Kishioka 1001-1, Suzuka, Mie, Japan

${ }^{2}$ Acupuncture Center, SUMS, Kishioka 1275-3, Suzuka, Mie, Japan

${ }^{3}$ ASSA Acupuncture and Orthopedic Clinic, Shiroko-2926, Suzuka, Mie, Japan ${ }^{4}$ Institute of Traditional Chinese Medicine, SUMS, Kishioka 1001-1, Suzuka, Mie, Japan

${ }^{5}$ Sourire Acupuncture Clinic, Nakasu-589, Ise, Mie, Japan

*Author for correspondence:

E-mail: zwp2000@suzuka-u.ac.jp 
of blood circulation [5]. As a result, the accumulated waste material appear associated with the stimulator of chronic dull pain and stiffness of the neck and shoulder [6].

In concurrence with the above survey results, shoulder and neck pain is also the leading major complaint occupying the ratio of $24 \%$ of the new patients in our attached acupuncture clinic, together with the same trend information that occurs most frequently in the female according to statistics from 2015 to 2016 [7]. However, the treatments for it varied according to acupuncturists by their styles and experiences until now. Most of the therapeutic methods followed with a western style by stimulating tender points and indurations on the local neck/ shoulder regions and interscapular areas. On the other hand, stiff shoulder is principally resulted from pathogenic wind-cold, deficiency of Liver-blood or blood stasis due to Qi stagnancy according to traditional Chinese diagnostic methods, so the general principles of treatment to relieve or improve their different pathogenic factors seem to be more effective and necessary. Since 2012, we have been intending to develop some more effective original standard approaches to acupuncture, for that reason we developed a protocol by means of planning treatment according to an overall analysis of patients' symptoms and signs, together with a pilot study to new patients with a chief complaint of shoulder stiffness aiming at examining its availability.

\section{Methods/Design}

\section{Objectives}

Thirty-two new patients from the attached acupuncture clinic of Suzuka University of Medical Science (SUMS), who complained of chronic dull pain and stiffness of the neck and shoulder without any arm symptoms, and those who were willing to participate in the experiments signed up as clinical research volunteers were recruited as participants of our study. All treatment procedures were submitted to the ethics Committee of the SUMS and were approved with the process number 2014-200.

\section{Intervention}

The test participants were allocated to one of three groups of Blood stasis due to Qi stagnancy (Group-1), Deficiency of Liver-blood (Group-2), Yin deficiency of Liver and Kidney (Group-3), according to their subjective symptoms and signs filled out in the existent detailed questionnaire of our clinic (Table 1). All three groups received acupuncture treatments once a week over two weeks in accordance with the following protocol. During the treatment period, participants were prohibited from receiving any concomitant treatments. Procedure and diagnose were performed by well-trained licensed acupuncturists, who have had more than five years of clinical acupuncture experience since completing a 4 year long college degree. In addition, the therapeutic effects were assessed by an independent researcher.

\section{Point selection}

In addition to the main points used as Table 1, we also

Table 1. Study Protocol of Acupuncture Treatment for Stiff Shoulder

\begin{tabular}{|c|c|c|c|}
\hline Types of Syndrome & Main Symptoms and Treatments & $\begin{array}{l}\text { Therapeutic } \\
\text { Principles }\end{array}$ & $\begin{array}{l}\text { Point } \\
\text { Prescription }\end{array}$ \\
\hline $\begin{array}{l}\text { Group-1 Blood Stasis due } \\
\text { to Qi Stagnancy }\end{array}$ & $\begin{array}{l}\text { Localized dull pain, distending pain and radiating neck/ } \\
\text { shoulder pain. Local twinge, Tenderness. Exacerbation } \\
\text { due to psychological factors. Pain relief during and after } \\
\text { movement, or when focusing their attention on something. } \\
\text { For those leading symptoms mainly due to Qi stagnancy } \\
\text { characterized with migratory pain, the same therapy will be } \\
\text { performed. }\end{array}$ & $\begin{array}{l}\text { Regulating Qi flow, } \\
\text { promoting blood } \\
\text { circulation and } \\
\text { removing blood } \\
\text { stasis }\end{array}$ & $\begin{array}{l}\text { LI-4, LR-3, SP-6, } \\
\text { GB-34 }\end{array}$ \\
\hline $\begin{array}{l}\text { Group-2 Deficiency of } \\
\text { Liver-blood }\end{array}$ & $\begin{array}{l}\text { Stiff shoulder gets worse when abusing their eyes, rigidity } \\
\text { on neck/shoulder. Always accompanied with eye fatigue } \\
\text { and mist. Relief by pressing. If blood deficiency is noted, } \\
\text { muscle jerking, rigidity or spasm (leg cramp) may also arise } \\
\text { even at other sites. }\end{array}$ & $\begin{array}{l}\text { Nourishing Liver- } \\
\text { blood }\end{array}$ & BL-18, SP-6, LR-3 \\
\hline $\begin{array}{l}\text { Group-3 Yin Deficiency of } \\
\text { Liver and Kidney }\end{array}$ & $\begin{array}{l}\text { Rigidity on neck/shoulder. Eye fatigue and mist, dry eye, } \\
\text { accompanied with symptoms like lassitude or pain on low } \\
\text { back and lower limbs. Relief by pressing. If Yin deficiency is } \\
\text { noticeable, symptoms like dysphoria and feverish sensation } \\
\text { in palms and soles are accompanied. While not noticeable, a } \\
\text { treatment likewise in the way of insufficiency of Kidney and } \\
\text { Liver will be performed. }\end{array}$ & $\begin{array}{l}\text { Nourishing Yin and } \\
\text { calming Liver }\end{array}$ & $\begin{array}{l}\text { BL-18, BI-23, SP-6, } \\
\text { KI-7, LR-3 }\end{array}$ \\
\hline
\end{tabular}


Table 2. Point Prescription according to Symptomatic Meridians

Taiyang Meridian (neck and shoulder) $\mathrm{SI}-3, \mathrm{BL}-64$ Yangming Meridian (anterior neck and shoulder) LI-4, ST-36 Shaoyang Meridian (lateral neck and shoulder) SJ-5, GB-34

Table 3. Local Point Prescription on Anatomical Sites

Anatomical Sites Point Prescription

Musculus semispinalis capitis $\quad$ BL-10

Musculus rhomboideus minor $\quad \mathrm{BL}-11$

Musculus supraspinatus $\quad \mathrm{SI}-12$

Musculus levator scapulae

SI-14

added some points according to symptomatic meridians (Table 2) on Taiyang, Yangming and Shaoyang channels, because on the basis of meridian theory, those three Yang meridians all go along the regions of back, shoulder and neck. In addition, acupoints mostly located near the pathological changes of the anatomical sites, such as BL-10 at semispinalis, BL-11 in rhomboid minor and splenius cervicis, SI-12 in supraspinatus muscle, and SI-14 in levator scapulae were used in all the objects aiming to remove obstruction induration in the associated channel and relax the related strained muscles ultimately (Table 3).

\section{Needling manipulation}

UNICO disposable stainless steel acupuncture needles with tubes $(\varphi 0.18 \times 48 \mathrm{~mm}$, Nisshin Medical Instrument Co., Osaka) were manually inserted ranging from $7 \mathrm{~mm}$ to $37 \mathrm{~mm}$ in depth according to the site of points, which were calculated referring the A-B Classic of Acupuncture and Moxibustion. Since stiff shoulder is mostly confused with syndromes of deficiency and excess, after the de qi sensation in traditional Chinese acupuncture, the needles were not manipulated definitely with any reinforcing and reducing methods, but were retained for 10 minutes.

\section{Evaluation}

The subjective magnitude of pain and stiffness on neck and shoulder sites was measured overall by visual analog scale (VAS), which is commonly used as the outcome measure for such studies. Its simplicity, reliability, and validity, as well as its ratio scale properties, make the VAS the optimal tool for describing pain severity or intensity [8].

\section{Statistical analysis}

All data were given as mean values \pm standard deviations in each group. Changes of all the participants over time were analyzed by paired t-tests. Within-group comparisons were evaluated by one-way analysis of variance with repeated measures followed by posthoc Tukey's multiple comparisons (SPSS Inc., Chicago, IL, USA). The levels of statistical significance were set at $\mathrm{p}<0.05$ and $\mathrm{p}<0.01$.

\section{Results}

Thirty-two patients attended at first, one patient in the Group-1 and two patients in the Group-3 dropped out of the study due to scheduling problems. Twentynine volunteers composed of two males and twentyseven females, with an average age of $70 \pm 16$ years old completed our trail study. Of all participants, 85.3\% had had complaints for more than six months. Besides some minor side effects like temporary post-needling soreness and distension left on the needling sites immediately after treatment, no adverse events were reported.

The averaged VAS values of all the participants at the baseline initial consultation decreased gradually after the treatments, which also showed a significant decrease at the third consultation when compared with the baseline VAS values $(\mathrm{p}<0.01)$ (Figure 1).

For the differentiation of symptoms, VAS scores in the Group-1 $(n=12)$ varied from 81 to 64 and 50 at the second and third consultation, which showed $21 \%$ and $38 \%$ decreased separately when compared with the baseline (Figure 2). The averaged VAS scores in Group-2 changed from 62 to 59 and 45 gradually, which showed $5 \%$ and $28 \%$ (vs baseline, $\mathrm{p}=0.05$ ) decrease as compared with that of baseline (Figure 3). The VAS values in Group-3 $(n=12)$ reduced from 68 to 54 and 45 , with a ratio change of $-17 \%$ and $-35 \%$ $(\mathrm{p}=0.053)$, respectively (Figure 4).

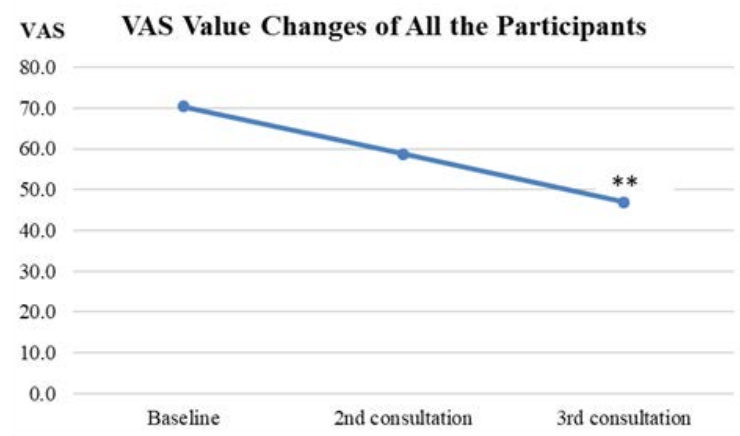

Figure 1. VAS value changes of all the participants. VAS values are expressed as the mean \pm standard deviation. The 2 nd and 3 rd consultations indicated the results of 1 st and 2 nd treatment respectively. ${ }^{*}$ Significantly different from that at baseline $(p<0.01)$. 


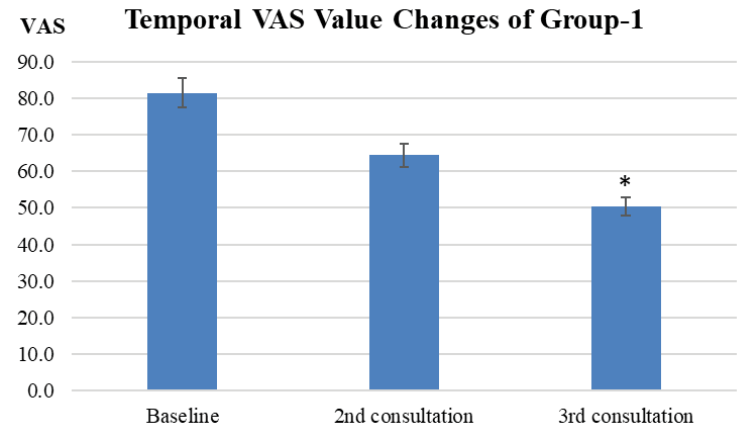

Figure 2. Temporal VAS value changes of Group-1. Values are expressed as the mean \pm standard deviation. The 2 nd and 3 rd consultations indicated the results of 1 st and 2nd treatment, respectively. *Significantly different from that at baseline $(p<0.05)$.

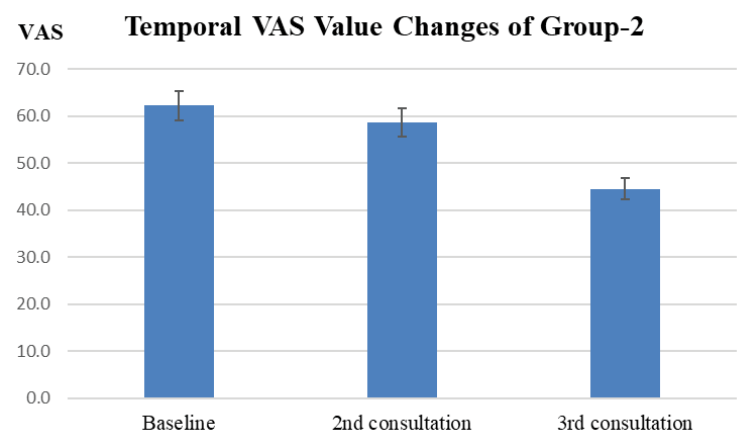

Figure 3. Temporal VAS value changes of Group-2. VAS values are expressed as the mean \pm standard deviation. The 2 nd and 3 rd consultations indicated the results of 1 st and 2 nd treatment respectively.

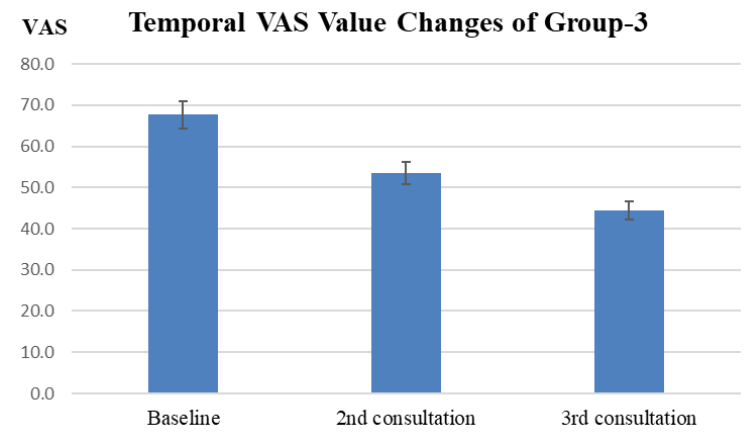

Figure 4. Temporal VAS value changes of Group-3. VAS values are expressed as the mean \pm standard deviation. The 2 nd and 3 rd consultations indicated the results of 1 st and 2 nd treatment respectively.

\section{Discussion}

The primary aim of the study was to develop an original protocol based on the theory of planning treatment according to diagnosis by TCM and try to standardize a therapeutic modality of stiff shoulder, which seems less attractive and non-mainstream in Japanese acupuncture therapy. In order to do this we divided syndromes into three groups, which are considered as the most common symptoms based upon patients' clinical findings and our experience in Japan.

Owing to the Three Yang Meridians of Hand travel along the lateral aspect of the upper extremities [9], where are exactly in accordance with the main complaining site of stiff shoulder patients. Therefore, acupoints on the meridians as referred to above are usually adopted to use by acupuncturists. In our protocol, we selected points on the base of differentiation of symptoms and signs (Table 1), symptomatic meridians and local symptomatic anatomical sites as well (Table 2 and Table 3 ), aiming to promote blood circulation, relax muscles and as a result of relieving dull and heavy pain appeared on the neck and shoulder [6].

During the experimental period, patients of Yin Deficiency of Liver and Kidney type (Group-3) consisted of the greatest numbers of the participants, which was possibly due to the mean age of patients [10]. Because deficiency of kidney essence and insufficiency of vital essence and energy are considered often to appear in seniors due to the wasting of the body in TCM, which is manifested as dizziness, lassitude in the loins and legs, eye fatigue, etc. [11]. As shown in Fig. 1 results, though the VAS changing trend differed among participants during the experimental period due to some reasons, the protocol appeared to be effective on the whole for improving and relieving the symptoms of stiff shoulder by varying degrees after two treatments without side effects.

For the effects of divided three groups, acupuncture therapy seemed to be all effective in relieving the complaints separately. Especially at the third consultation, Group-1 showed a significant decrease than the baseline probably on account of those more easily relieved excess syndromes. Moreover, Group-2 and Group-3 showed almost significant than the baseline $(\mathrm{p}=0.05)$ and a likely prospect would be presumed if there were many more participants. From these changes in VAS score, we conclude that this protocol would provide an effective medical treatment for stiff neck and shoulder.

There are still several limitations in the present study. Firstly, participants ranged in age from 23 to 86 years old, the great difference of their body-builds might lead to discordancy in advantageous effect changes. A rather confined age of participants would be more appropriate to make a valuation of protocol. Secondly, several practitioners engaged the acupuncture procedure in this study, since any minute manipulation difference might affect the therapeutic action, only 
one or fewer practitioners in the study was required as an ideal condition. Thirdly, this study was conducted and prolonged for over two years, even though we tried to maintain the room temperature at $26^{\circ} \mathrm{C}$ in the acupuncture clinic as possible, different seasons and unexpected air temperature changes might influence the patient's complaints and evaluation of effects, which would probably lead to a discordancy. Therefor shortening the whole experimental period into a same season would be a suggested improvement in a future study. Finally, we conducted the trial study in parallel with their treatment in our clinic, in view of the real experimental condition that how many times the patients could continue easily, we only determined therapeutic effects twice over and failed to evaluate the therapeutic action for longer periods for time. While mercifully, the trial study is still in progress currently, we will continue to report the validity and availability of the protocol after increasing more cases and extending the experimental and follow-up periods in a new stage.

In conclusion, these results indicate that the present study protocol characterized with planning treatment according to diagnosis would be effective in relieving the dull pain and stiffness on neck and shoulder regions, and it would be appropriate as a standard, effective and safe medical treatment for stiff neck and shoulder.

\section{Acknowledgement}

The authors would like to thank Professor Mark LaForge for revising the English in this manuscript.

\section{Executive summary}

Background: In order to standardize an effective acupuncture therapy for chronic dull pain and stiffness of neck and shoulder, we made a protocol characterized with planning treatment according to diagnosis and examined its availability.

Methods and findings: Thirty-two patients were categorized into three groups based on the pathophysiology of Traditional Chinese Medicine: Blood stasis due to Qi stagnancy (Group-1), Deficiency of Liver-blood (Group-2), Yin deficiency of Liver and Kidney (Group-3) according to their subjective symptoms and signs. Acupuncture treatment was performed once a week over a two week period in accordance with the protocol that was arranged to each group. The averaged VAS values after the $3^{\text {rd }}$ therapy in the whole groups decreased significantly $(p<0.01)$ as compared with that of the initial visit before therapy. Investigating each group, the averaged VAS value after the $3^{\text {rd }}$ therapy in Group 1 was significantly $(p<0.05)$ lower than that of the initial visit at baseline. In other groups, although the VAS scores showed a gradual decrease according to the treatment, there was unfortunately no significance in the changes of the VAS. There were no side effects in all the treatment of this study.

Conclusions: These results indicate that the present study protocol would be effective in relieving the patient's subjective symptoms, and it would alternatively provide an effective and safe medical treatment for stiff neck and shoulder, although it should be modified for Groups 2 and 3.

\section{References}

1. Lumime J, Koes B, Hendriksen I. Prevalence and incidence of shoulder pain in the General population: a systematic review. Scand J of Rheum 33: 73-81 (2004).

2. Minister's Secretariat, Ministry of Health, Labour and Welfare. Graphical Review of Japanese Household: From Comprehensive Survy of Living Conditions 30: (2013).

3. Nabeta T, Kawakita K. Relief of chronic neck and shoulder pain by manual acupuncture to tender points - a sham-controlled randomized trial. Comp Therap Med 10: 217-227 (2002).

4. Dolder PA, Ferreira PH, Refshauge KN. Effectiveness of soft tissue massage and exercise for the treatment of non-specific shoulder pain: a systematic review with meta-analysis. Br J Sports Med 1-12: (2012).

5. Nishijyo K. Scientific Appraisal of Clinical Acupuncture. Ishiyaku Publishers, Inc., Tokyo, Japan, 112-121 (2000).
6. Sasaki K. Needling Treatment of Stiff Shoulder with Western Medicine. The Japanese Journal of Acupunct Manual Therap 65: 30-34 (2006).

7. Ishiyama R, Kanehara M, Nishimura K. Patient Investigation at first medical examination in Acupuncture Clinic of SUMS. Collection of Abstracts of $32^{\text {nd }}$ Midland Local Congress of JSAM. November 16: (2014).

8. Katz J, Melzack R. Measurement of pain. Surg Clin North Am 79: 231-252 (1999).

9. Liu GW, Cao LY. A Complement Work of Present Acupuncture and Moxibustion: Acupoints \& Meridians, 1st ed. Huaxia Publishing House 3-5: (1997)

10. Liu GW, Acupuncturology. Oriental Medicine Publishing House, 52 : 155-157 (2000).

11. Yuan YX, Ren JX, Huang L. Chinese-English Dictionary of Traditional Chinese Medicine. $1^{\text {st }}$ ed. People's Medical Publishing House, Beijing, China, 601-602: (1997). 